\title{
Canine renal amyloidosis: A case report
}

\author{
BARBARA SZCZEPANKIEWICZ, URSZULA PASŁAWSKA, MACIEJ GRZEGORY, \\ PAWE $ヒ$ JONKISZ, PAULINA BORECKA*, MAGDALENA MARZEC*, \\ MAGDALENA BRZOZOWSKA**, MARCIN NOWAK*
}

\begin{abstract}
Department of Internal Medicine and Clinic of Diseases of Horses, Dogs and Cats, Faculty of Veterinary Medicine, Wrocław University of Environmental and Life Sciences, PI. Grunwaldzki 47, 50-375 Wrocław *Department of Pathology, Faculty of Veterinary Medicine,

Wrocław University of Environmental and Life Sciences, ul. C. K. Norwida 31, 50-375 Wrocław **Department and Clinic of Veterinary Surgery, Faculty of Veterinary Medicine, Wrocław University of Environmental and Life Sciences, PI. Grunwaldzki 51, 50-366 Wrocław
\end{abstract}

\section{Szczepankiewicz B., Pasławska U., Grzegory M., Jonkisz P., Borecka P., Marzec M., Brzozowska M., Nowak M. \\ Canine renal amyloidosis: A case report \\ Summary}

Amyloidosis is a disease caused by the deposition of amorphous extracellular protein, leading to impaired kidney function. Canine and feline amyloidosis is associated with the deposition of AA protein. The disease is hereditary and is related to breed but not sex. Predisposed breeds include the Shar Pei dog and Abyssinian cat. Proteinuria resulting in hypoalbuminemia due to changes in renal glomeruli is the first clinical sign. In addition, a decreased appetite, anorexia, vomiting, lethargy, polyuria and polydipsia may be observed. In order to diagnose the disease, serum amyloid A levels may be measured. However, a definitive diagnosis is made on the basis of an intravital renal biopsy and the presence of amyloid in the histopathological examination. The main goal of treatment is to reduce inflammation and proteinuria. If nephrotic syndrome occurs, the prognosis is guarded to poor, and the majority of patients do not survive one year. The definitive diagnosis is based on the post-mortem examination, in which the presence of amyloid deposits is confirmed in the kidney tissue. We present the case of a 7-year-old female Shar Pei diagnosed with kidney amyloidosis, on the basis of which we have developed a prevention scheme for clinical practice.

Keywords: amyloidosis, kidney, proteinuria, Familial Shar-Pei Fever

The purpose of this case report is to present the correct diagnosis and latest treatment of suspected renal amyloidosis in the dog. The first report of a disease resembling generalised amyloidosis goes back to the 1600s. In medicine, the term "amyloidosis" was coined in 1854. It was named after amyloid, which deposited in tissues and (similarly to starch) stained blue with iodine. Currently, at least 25 human and eight animal amyloid precursor proteins are known.

Amyloidosis (betafibrosis) is caused by an extracellular deposition of amorphous fibrillary protein aggregates. The structure of the proteins that form amyloid fibers varies. However, they all form a common secondary structure known as the beta-pleated sheet. The spatial arrangement of amino acids in the amyloid polypeptide chain resembles a sheet of paper folded into a harmonica shape. Amyloid is composed of protein fibers that form structures maintained by hydrogen bonds. Glycosaminoglycans and a specific glycoprotein known as the amyloid $\mathrm{P}$ component are also linked to the protein fibers (4).

In the electron microscope, all amyloid proteins form a regular linear structure of unbranched fibers 7.5-10 $\mathrm{nm}$ in diameter. Under the light microscope, amyloid stained with hematoxylin and eosin forms homogenous pink extracellular deposits that resemble cotton wool or blotches. After staining with Congo red, the extracellular deposits are orange and show light green birefringence under polarized light (14).

In cats and dogs, amyloidosis is caused mainly by the amyloid A protein. Amyloid A is a fragment of serum amyloid $A$, an acute phase protein, which is produced in numerous tissues, mainly in the liver. In the presence of inflammatory mediators such as IL-1, IL- 6 and TNF- $\alpha$, SAA mRNA increases up to 1000-fold. Serum amyloid A affects neutrophil hemotaxis, stimulates degranulation and phagocytosis as well as the release of cytokines (1). 
In human medicine, amyloidosis can be primary or secondary. Primary amyloidosis is associated with light-chain monoclonal gammopathy, while secondary, reactive amyloidosis occurs in inflammatory, neoplastic and rheumatic diseases, as well as in juvenile arthritis. Reactive amyloidosis may also be idiopathic. In veterinary medicine, there is also a familial form of the disease in Shar Pei dogs and Abyssinian cats, which is classified as secondary (reactive) amyloidosis $(1,11)$.

Cats and dogs are predisposed to renal amyloidosis, which leads to chronic renal failure. In dogs, renal amyloidosis affects mainly glomeruli, causing proteinuria and, in effect, hypoalbuminemia (15). The accumulation of amyloid depositions does not always reflect the degree of proteinuria, as amyloid may accumulate in interstitial tissue and renal papillae (13). Amyloid may also accumulate in other organs, such as the spleen, liver, adrenal glands, heart, thyroid gland, prostate glands and lymph nodes.

\section{Case report}

A seven-year-old female Shar Pei dog was presented to the Department of Internal Diseases with Clinic for Horses, Dogs and Cats with a history of weight loss and reduced physical activity. The owner also reported episodic fever. The dog had been previously treated for pancreatitis and cystitis. Skin mucinosis was also observed.

The clinical examination revealed a normal physical appearance and body condition. The body temperature, pulse and respiratory rate of the dog were within reference ranges. The oral examination showed the presence of tartar without gingivitis or gingival erosions. Jaw, mandibular and neck pain were excluded as the cause of decreased appetite. All palpable lymph nodes were normal.

The complete blood count and blood biochemistry were within reference ranges. The patient was given analgesics, and blood tests were recommended after 3-4 weeks.

The patient returned for a re-examination due to periarticular tarsal swelling. The clinical examination revealed abdominal pain and a temperature of above $40 \cdot 1^{\circ} \mathrm{C}$. The blood tests showed an elevated serum creatinine level of $200 \mathrm{umol} / \mathrm{l}$ (reference range from $88 \mathrm{umol} / \mathrm{l}$ to $150 \mathrm{umol} / \mathrm{l}$ ).

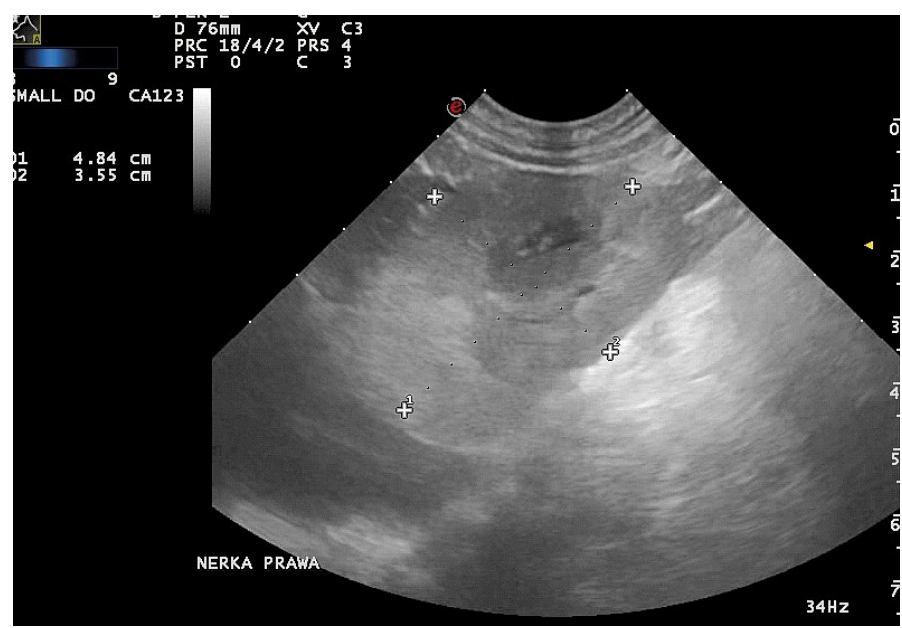

Fig. 1. Ultrasound image of right kidney
The remaining blood parameters were within reference ranges. The systolic blood pressure was within the normal range. The physicochemical analysis of urine revealed an increased urine protein to creatinine (UPC) ratio of 1.24 (reference range in dogs 0-0.6). SAA was within the reference range.

A low protein, sodium and phosphorus diet for dogs with chronic renal failure was implemented. In addition, rhuzone (Rheum palmatum) with anti-fibrotic properties was employed for renal protection and to slow down the progression of renal failure. Colchicine, an alkaloid that decreases the incidence of episodes of familial Shar Pei fever and inhibits the production of serum amyloid A, was also administered. The treatment was introduced to limit the formation of amyloid. Colchicine also stabilizes the glomerular filtration rate in patients with proteinuria.

The patient appeared stable during the four-week observation period, and there were no episodes of health decline or fever. Blood and urine biochemistry revealed a slight decrease in the serum creatinine concentration and an unchanged UPC. The owners were informed that the dog should receive specialised care because of the proteinuria.

In the following four-week period, the clinical condition of the dog worsened. The animal was apathetic and started vomiting. The complete blood count and biochemistry were within reference ranges, while the UPC increased significantly to 4 units. The abdominal ultrasound showed reduced round kidneys, loss of the corticomedullary border, as well as an increase in the thickness and echogenicity of the renal cortex. There was no calyceal or renal pelvic widening. The renal capsule was smooth (Fig. 1 and 2). The ultrasound examination did not reveal any structural changes in the remaining organs of the pelvic and abdominal cavity. Telmisartan and omeprazol were introduced to decrease proteinuria and to protect the gastric mucosa, respectively.

In the following three weeks, the UPC decreased to 1.1. Subsequent urine examinations every 3-4 weeks revealed stable proteinuria. However, the clinical condition of the dog deteriorated. The dog continued to be apathetic, had no appetite and continued to lose weight. The owners opted for euthanasia.

The post-mortem examination revealed macroscopic lesions typical of amyloidosis. The kidneys were yellowish-

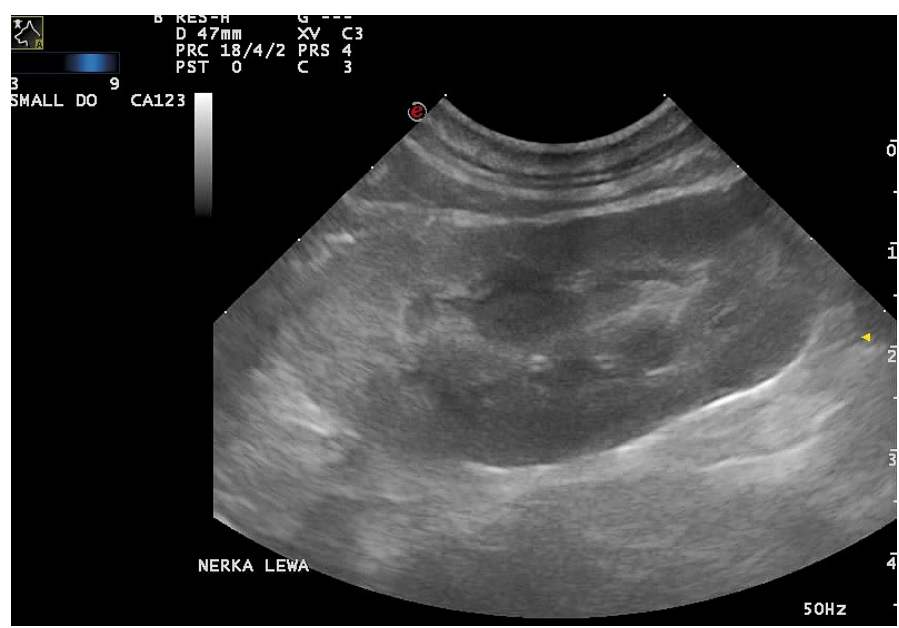

Fig. 2. Ultrasound image of left kidney 


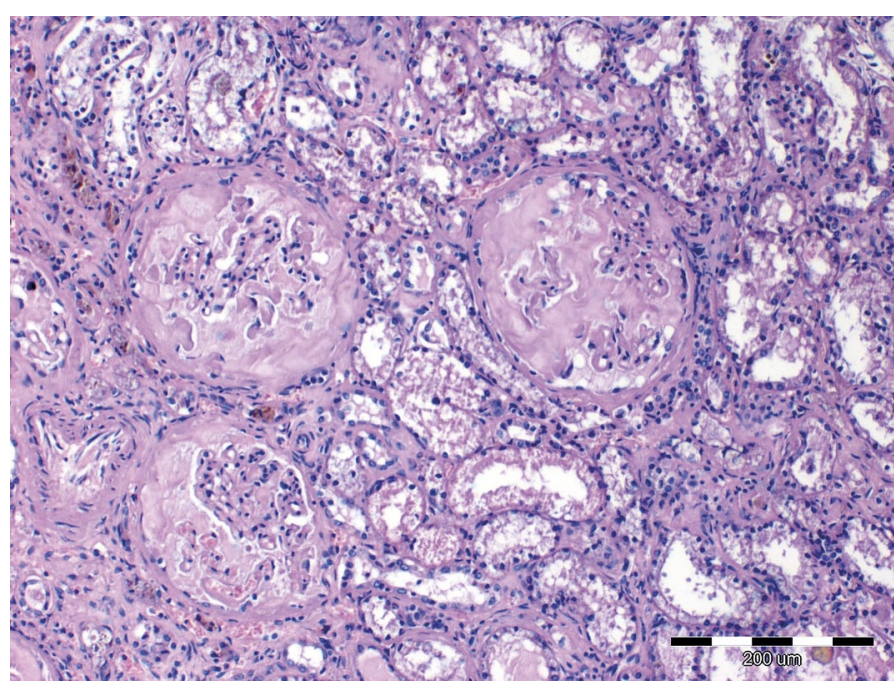

Fig. 3. Histopathological examination of kidneys, H\&E stain. Visible amyloid deposition in glomeruli. Magnification $\times 10$

grey and small (Fig. 3). Specimens of the kidney lesions were collected and stained with $\mathrm{H} \& \mathrm{E}$ and Congo red. The histopathological examination showed amyloid depositions in the glomeruli and interstitial tissue and, to a lesser extent, in the vessel walls of large arteries. In addition, there were numerous lymphocytic infiltrates, suggesting the presence of an accompanying inflammatory process. Furthermore, there were degenerative lesions in epithelial cells in the renal cortical layer (Fig. 3, 4).

\section{Discussion}

Renal amyloidosis is a hereditary disease in the Shar Pei, with a prevalence of $23 \%$ in the USA (11). Data suggest that renal amyloidosis affects $37 \%$ of dogs with proteinuria (3). In Poland there are no data on this subject.

The most common symptoms of the disease are a decrease or lack of appetite, vomiting, apathy, polyuria and polydipsia, as well as haematuria. In the case of proteinuria exceeding $3.5 \mathrm{~g} /$ day, there can be other signs of the nephrotic syndrome, such as peripheral oedema and transudates into the pleural and abdominal cavity. In the first stage of the disease, kidneys affected by amyloidosis may appear enlarged in the ultrasound examination because of an inflammatory infiltrate. In the subsequent stages of the disease, the kidneys may be reduced in size $(11,15)$. The progression of the disease depends on the location and extent of kidney damage caused by amyloid deposition. In addition to the general signs of the disease, organ-specific or systemic signs may be observed.

Diagnosing amyloidosis is challenging. Measuring serum amyloid $\mathrm{A}$, which is considered a reliable marker of an inflammatory process, similarly to CRP, may be useful. Serum amyloid A may be measured both in dogs and cats $(2,10)$ in blood serum (sSAA) or urine (uSAA). However, it is not an amyloidosis-specific marker. Serum amyloid A may also increase in the course of other systemic diseases, such as renal failure,

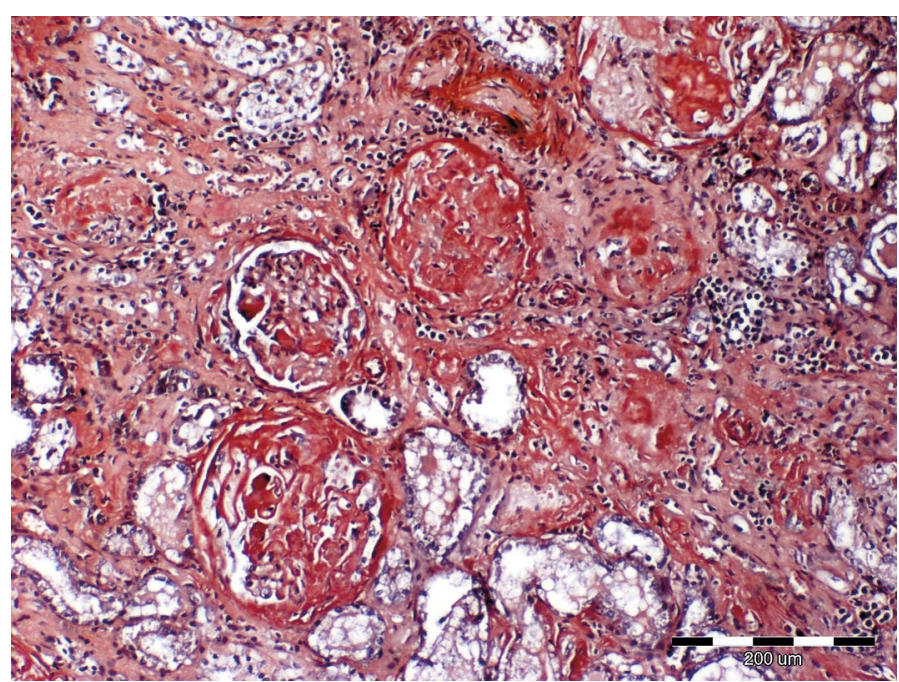

Fig. 4. Histopathological examination of kidneys, Congo red stain. Magnification $\times 10$

diabetes or neoplasia (9). To date, there are no clear guidelines for assessing the disease progression. No clear relationship has been found between SAA levels and the degree of proteinuria, which would facilitate assessment of the progression of the disease $(5,8)$. A definitive diagnosis of renal amyloidosis is based on the presence of amyloid in kidney samples in the histopathological examination. The samples can be collected intravitally or post-mortem. Intravital samples may be collected by an ultrasound-guided renal biopsy. However, this technique is invasive. In addition, it may not be diagnostic in some dogs (particularly the Shar Pei) in which amyloid is deposited in the renal medulla. Sections of 8-10 microns in thickness are cut from paraffin-embedded blocks, as thinner sections may fail to stain. Amyloid depositions are visible in the form of amorphous masses after standard H\&E staining. Hence, additional stains for amyloid, such as Congo red, can be used. In the histopathological examination, amyloid depositions are visible in the glomeruli, the renal medulla or the vessel walls of arteries or arterioles. Congo red stains the amyloid depositions reddish-orange. The sections may also be evaluated using polarizing light microscopy in order to visualize willow-green amyloid birefringence. Immunohistochemistry, immunofluorescence or genetic studies may be carried out to determine the kind of amyloid deposits.

Once amyloidosis has been diagnosed, appropriate treatment, aimed at minimising the inflammatory response and limiting proteinuria, should be administered. Colchicine decreases hepatic SAA production and may be administered at $0.01-0.03 \mathrm{mg} / \mathrm{kg}$ p.o. every 24 hours. The adverse effects of colchicine administration include gastrointestinal disorders. Alternatively, dimethyl sulfoxide (DMSO, $90 \mathrm{mg} / \mathrm{kg}$ p.o. three times a week or subcutaneously diluted to $1: 4$ with saline) may be used. DMSO has an anti-inflammatory effect, reduces interstitial fibrosis and may improve kidney 
function and reduce proteinuria. However, it may cause nausea and reduce appetite (12). Hence, methylsulfonylmethane (MSM) may be used as an alternative.

Telmisartan, a selective angiotensin II AT receptor antagonist is also used to treat amyloidosis. It acts by decreasing blood pressure and proteinuria in animals with chronic renal failure. Its efficacy in reducing proteinuria has been confirmed in dogs and cats (11). The initial dose is $1 \mathrm{mg} / \mathrm{kg}$ SID p.o. and, if necessary, can be gradually increased to $3 \mathrm{mg} / \mathrm{kg}$ SID and then $3 \mathrm{mg} / \mathrm{kg}$ BID $(1,7)$.

Antithrombotic agents may also be administered, as the amyloid that deposits in the tunica media of arterial walls weakens them, making them brittle. Fibrin molecules accumulate around the bleeding site to form a thrombus. In this process, thrombosis is not autoregulated, because of the concurrent angiopathy (amyloid or hypertensive), which inhibits vessel contraction, leading to the formation of large-sized thrombi $(7,16)$. Acetylsalicylic acid (ASA) is the most common antithrombotic drug. It is used in small doses (1-5 mg/kg p.o. every $24 \mathrm{~h}$ ) in case of hypercoagulability $(1,6)$. All non-steroidal anti-inflammatory drugs, including acetylsalicylic acid (ASA), damage gastrointestinal mucosa by decreasing the production of prostaglandins through inhibiting the activity of type 1 cyclooxygenase (COX-1). In addition, they inhibit the function of platelets, causing bleeding. Their antiplatelet effect affects angiogenesis. It also delays the healing of gastric erosions and ulcers caused by other drugs or a high serum urea level. Therefore, the risk of ulcer formation needs to be contemplated when choosing antithrombotic treatment. Hence, the use of drugs preventing gastrointestinal damage, such as omeprazole, should be considered. The recommended dose of omeprazole is $1-2 \mathrm{mg} / \mathrm{kg}$ p.o. every 24 hours.

Urine should be examined every 2 to 3 weeks. The dosage of drugs depends on the clinical state of the patient and the degree of proteinuria. Many animals develop the nephrotic syndrome due to persistent proteinuria. In such cases, the prognosis is guarded to poor. Those animals usually die within a year of nephrotic syndrome diagnosis, and only $8.5 \%$ survive over one year (4).

The definitive diagnosis is based on the post-mortem examination of renal tissue specimens. The postmortem examination reveals lesions in other organs. Macroscopically, the kidneys are greyish-yellow and contain pale yellow glomeruli on cross section.

In conclusion, amyloidosis is a severe, genetically determined disease often leading to the death of the affected animals. Therefore, prophylactic tests should be developed for early diagnosis of the disease so as to eliminate the affected animals from breeding. A thorough medical history should be collected from the owners of the dogs predisposed to the disease in order to detect transient fevers and periarticular swelling. The owners should be advised to carry out routine blood and urine tests. It would also be useful to carry out prophylactic renal ultrasound examinations. In selected cases, it may be necessary to carry out renal biopsies. The proposed diagnostic regimen may facilitate early diagnosis of amyloidosis in animals.

\section{References}

1. Bugbee A., Coleman A., Wang A., Woolcock A., Brown A.: Telmisartan of refractory proteinuria in dogs. J. Vet. Intern. Med. 2014, 28, 1871-1874.

2. Ceron J., Eckersall P., Martinez-Subiela S.: Acute phaseprotein in dogs and cats: Current knowledge and future perspectives. Vet. Clin. Pathol. 2005, 34, 85-99.

3. Cook A., Cowgill L.: Clinical and pathological features of protein-losing glomerular disease in the dog: a review of 137 cases (1985-1992). J. Am. Anim. Hosp. Assoc. 1996, 32, 313-322.

4. DiBartola S., Benson M.: The pathogenesis of reactive systemic amyloidosis. J. Vet. Intern. Med. 1989, 3, 31-41.

5. DiBartola S., Tarr M., Benson M.: Tissue distribution of amyloid deposits in Abyssinian cats with familial amyloidosis. J. Comp. Pathol. 1986, 96, 387-398.

6.Dudley A., Thomason J., Fritz S., Grady J., Stokes J., Wills R., Pinchuk L., Mackin A., Lunsford K.: Cyclooxygenase expression and platelet function in healthy dogs receiving low-dose aspirin. J. Vet. Intern. Med. 2013, 27, 141-149.

7. Jull B.: Zakrzepica płucna i amyloidoza kłębuszków nerkowych u psa Weterynaria po Dyplomie 2000, 1, 66-67.

8. Lees G., Brown S., Elliott J., Grauer G., Vaden S.: Assessment and management of proteinuria in dogs and cats: 2005 ACVIM forum consensus statement (small animal). J. Vet. Intern. Med. 2005, 19, 377-385.

9. Littman M.: Protein-losing nephropathy in small animals. Vet. Clin. North. A. Small Anim. Pract. 2011, 41, 40-42.

10. Paltrinieri S.: The feline acute phase reaction. Vet. J. 2008, 177, 26-35.

11. Pressler B., Vaden S.: Managing renal amyloidosis in dogs and cats. Vet. Med. 2003, 98, 320-323.

12. Sasaki K., Ma Z., Khatlani T., Okuda M., Inokuma M., Inishi T.: Evaluation of feline serum amyloid A (SAA) as an inflammatory marker. J. Vet. Med. Sci. 2003, 65, 545-548

13. Segev G., Cowgill L., Jessen S., Berkowitz A., Mohr A., Aroch I.: Renal Amyloidosis in Dogs: A Retrospective Study of 91 Cases with Comparison of the Disease between Shar-Pei and Non-Shar-Pei Dogs. Vet. Intern. Med. 2012, 26, 259-268

14.Shtrasburg S., Gal R., Gruys E., Perl S., Martin B., Kaplan B., Koren R., Nyska A., Pras M., Livneh A.: An ancillary tool for the diagnosis of amyloid A amyloidosis in a variety of domestic and wild animals. Amer. Coll. Vet. Path. 2005, 42, 132-139.

15. Slauson D., Gribble D., Russell S.: A clinicopathological study of renal amyloidosis in dogs. J. Comp. Pathol. 1970, 80, 335-343.

16. Veglio F., Paglieri C., Rabbia F., Bisbocci D., Bergui M., Cerrato P.: Hypertension and cerebrovascular damage. Atheroscler. 2009, 205, 331-341.

Corresponding author: Barbara Szczepankiewicz, DVM; e-mail: barbara.szczepankiewicz@upwr.edu.pl 